\title{
Crystal structure of $\left[1 R^{*}, 2 S^{*}, 3 R^{*}\left(S^{*}\right), 4 S^{*}\right]-\alpha-0 \times 0-3-(10-0 \times 0-9-p h e n y l-$ bicyclo[6.2.0]-dec-1(8)-en-9-ylbicyclo[2.2.1] heptane-2-acetic acid methyl ester, $\mathrm{C}_{4} \mathrm{O}\left(\mathrm{C}_{6} \mathrm{H}_{12}\right)\left(\mathrm{C}_{6} \mathrm{H}_{5}\right)\left[\mathrm{C}_{7} \mathrm{H}_{10}\left(\mathrm{C}_{3} \mathrm{H}_{3} \mathrm{O}_{3}\right)\right]$
}

\author{
K. Peters, E.-M. Peters
}

Max-Planck-Institut für Festkörperforschung. Heisenbergstraße 1. D-70506 Stuttgart, Germany

J. Hegmann and M. Christl

Universität Würzburg, Institut für Organische Chemie, Am Hubland, D-97074 Würzburg, Germany

Received May 7, 1998, CSD-No. 409308

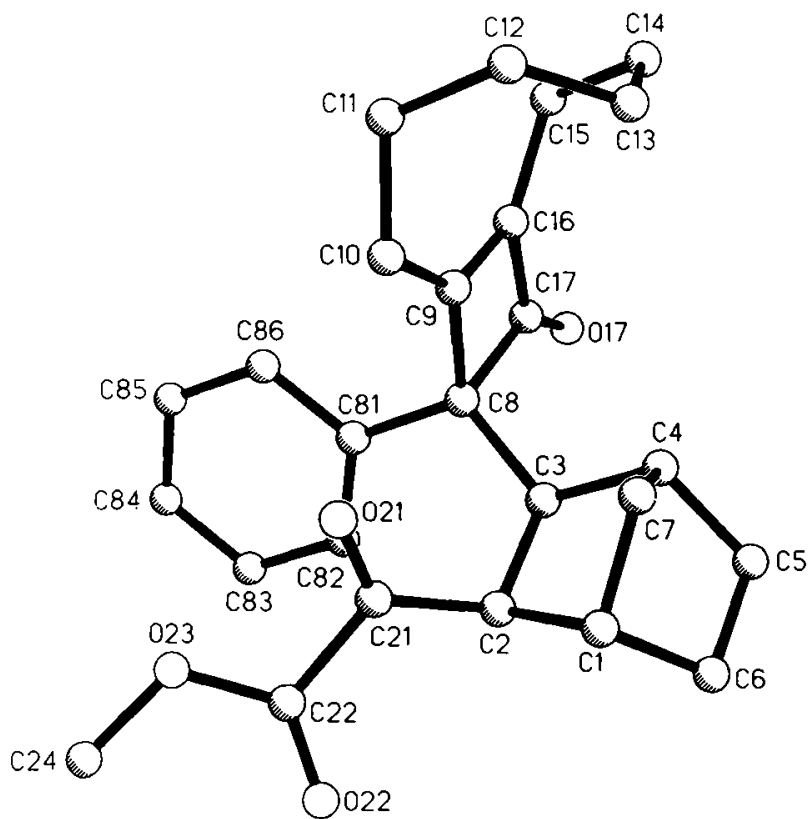

Source of material: The title compound was prepared according to ref. 1.

$\mathrm{C}_{26} \mathrm{H}_{3} 0 \mathrm{O}_{4}$, triclinic, $P \overline{1}$ (No. 2), $a=10.611(2) \AA, b=10.635(2) \AA$, $c=11.528(2) \AA, \alpha=96.95(3)^{\circ}, \beta=112.57(3)^{\circ}, \gamma=110.79(3)^{\circ}$, $V=1072.1 \AA^{3}, Z=2, R(F)=0.052, R_{\mathrm{W}}(F)=0.049$.
Table 1. Parameters used for the $X$-ray data collection

\begin{tabular}{|c|c|}
\hline $\begin{array}{l}\text { Crystal: } \\
\text { Wavelength: }\end{array}$ & $\begin{array}{l}\text { colorless plate, size } 0.4 \times 0.85 \times 0.25 \mathrm{~mm} \\
\text { Mo } K_{\alpha} \text { radiation }(0.71073 \AA)\end{array}$ \\
\hline$\mu:$ & $0.80 \mathrm{~cm}^{-1}$ \\
\hline Diffractometer: & Nicolet P3 \\
\hline Scan mode: & Wyckoff \\
\hline $\mathbf{T}_{\text {measurement: }}$ & $293 \mathrm{~K}$ \\
\hline $2 \theta_{\max }$ & $55^{\circ}$ \\
\hline $\mathrm{N}(h k)_{\text {unique: }}$ & 3904 \\
\hline Criterion for $F_{0}$ : & $F_{0}>3 \sigma\left(F_{0}\right)$ \\
\hline $\mathbf{N}(\text { param })_{\text {refined: }}$ & 272 \\
\hline Program: & SHELXTL-plus \\
\hline
\end{tabular}

Table 2. Final atomic coordinates and displacement parameters (in $\AA^{2}$ )

\begin{tabular}{|c|c|c|c|c|c|}
\hline Atom & Site & $x$ & $y$ & $z$ & $U_{\text {iso }}$ \\
\hline$H(1)$ & $2 i$ & $0.2676(3)$ & $0.0268(2)$ & $-0.3677(2)$ & 0.08 \\
\hline$H(2)$ & $2 i$ & $0.4160(2)$ & $0.2988(2)$ & $-0.2762(2)$ & 0.08 \\
\hline$H(3)$ & $2 i$ & $0.3897(2)$ & $0.3770(2)$ & $-0.1043(2)$ & 0.08 \\
\hline $\mathbf{H}(4)$ & $2 i$ & $0.2173(2)$ & $0.1738(2)$ & $-0.0566(2)$ & 0.08 \\
\hline$H(5 A)$ & $2 i$ & $0.4919(3)$ & $0.2359(3)$ & $0.0085(2)$ & 0.08 \\
\hline$H(5 B)$ & $2 i$ & $0.3956(3)$ & $0.0803(3)$ & $-0.0016(2)$ & 0.08 \\
\hline$H(6 A)$ & $2 i$ & $0.5214(3)$ & $0.1595(3)$ & $-0.1633(2)$ & 0.08 \\
\hline$H(6 B)$ & $2 i$ & $0.4283(3)$ & $0.0038(3)$ & $-0.1714(2)$ & 0.08 \\
\hline$H(7 A)$ & $2 i$ & $0.0768(3)$ & $0.0435(2)$ & $-0.2976(2)$ & 0.08 \\
\hline $\mathrm{H}(7 \mathrm{~B})$ & $2 i$ & $0.1476(3)$ & $-0.0472(2)$ & $-0.2227(2)$ & 0.08 \\
\hline$H(10 A)$ & $2 i$ & $-0.1151(2)$ & $0.2431(3)$ & $-0.4050(2)$ & 0.08 \\
\hline $\mathrm{H}(10 \mathrm{~B})$ & $2 i$ & $-0.1027(2)$ & $0.1094(3)$ & $-0.3679(2)$ & 0.08 \\
\hline$H(11 A)$ & $2 i$ & $-0.2584(3)$ & $0.2279(3)$ & $-0.2827(3)$ & 0.08 \\
\hline $\mathrm{H}(11 \mathrm{~B})$ & $2 i$ & $-0.3432(3)$ & $0.1175(3)$ & $-0.4223(3)$ & 0.08 \\
\hline$H(12 A)$ & $2 i$ & $-0.3239(3)$ & $-0.0559(3)$ & $-0.3364(3)$ & 0.08 \\
\hline$H(12 B)$ & $2 i$ & $-0.3826(3)$ & $0.0207(3)$ & $-0.2591(3)$ & 0.08 \\
\hline$H(13 A)$ & $2 i$ & $-0.2070(3)$ & $-0.0459(3)$ & $-0.1236(3)$ & 0.08 \\
\hline H(13B) & $2 i$ & $-0.0852(3)$ & $0.0577(3)$ & $-0.1550(3)$ & 0.08 \\
\hline$H(14 A)$ & $2 i$ & $-0.2129(3)$ & $0.1423(3)$ & $-0.0097(3)$ & 0.08 \\
\hline$H(14 B)$ & $2 i$ & $-0.0474(3)$ & $0.1550(3)$ & $0.0509(3)$ & 0.08 \\
\hline$H(15 A)$ & $2 i$ & $-0.0094(3)$ & $0.3755(3)$ & $0.0539(2)$ & 0.08 \\
\hline $\mathrm{H}(15 \mathrm{~B})$ & $2 i$ & $-0.1479(3)$ & $0.3257(3)$ & $-0.0858(2)$ & 0.08 \\
\hline$H(24 A)$ & $2 i$ & $0.1877(4)$ & $0.4327(4)$ & $-0.7311(3)$ & 0.08 \\
\hline$H(24 B)$ & $2 i$ & $0.2744(4)$ & $0.3388(4)$ & $-0.7219(3)$ & 0.08 \\
\hline$H(24 C)$ & $2 i$ & $0.3535(4)$ & $0.4873(4)$ & $-0.6185(3)$ & 0.08 \\
\hline$H(82)$ & $2 i$ & $0.4396(2)$ & $0.5161(2)$ & $-0.2104(2)$ & 0.08 \\
\hline H(83) & $2 i$ & $0.4870(3)$ & $0.6989(2)$ & $-0.3063(2)$ & 0.08 \\
\hline H(84) & $2 i$ & $0.3084(3)$ & $0.7859(3)$ & $-0.3871(2)$ & 0.08 \\
\hline $\mathrm{H}(85)$ & $2 i$ & $0.0787(3)$ & $0.6859(3)$ & $-0.3800(2)$ & 0.08 \\
\hline$H(86)$ & $2 i$ & $0.0286(3)$ & $0.5004(2)$ & $-0.2888(2)$ & 0.08 \\
\hline
\end{tabular}


Table 3. Final atomic coordinates and displacement parameters (in $\AA^{2}$ )

\begin{tabular}{|c|c|c|c|c|c|c|c|c|c|c|}
\hline Atom & Site & $x$ & $y$ & $z$ & $U_{11}$ & $U_{22}$ & $U_{33}$ & $U_{12}$ & $U_{13}$ & $U_{23}$ \\
\hline$C(1)$ & $2 i$ & $0.2904(3)$ & $0.0854(2)$ & $-0.2853(2)$ & $0.069(2)$ & $0.045(1)$ & $0.047(1)$ & $0.030(1)$ & $0.023(1)$ & $0.007(1)$ \\
\hline$C(2)$ & $2 i$ & $0.3178(2)$ & $0.2381(2)$ & $-0.2868(2)$ & $0.041(1)$ & $0.043(1)$ & $0.039(1)$ & $0.018(1)$ & $0.018(1)$ & $0.010(1)$ \\
\hline$C(3)$ & $2 i$ & $0.2989(2)$ & $0.2948(2)$ & $-0.1653(2)$ & $0.037(1)$ & $0.032(1)$ & $0.035(1)$ & $0.0141(9)$ & $0.0150(9)$ & $0.0093(9)$ \\
\hline$C(4)$ & $2 i$ & $0.2623(2)$ & $0.1665(2)$ & $-0.1136(2)$ & $0.051(1)$ & $0.043(1)$ & $0.047(1)$ & $0.022(1)$ & $0.022(1)$ & $0.019(1)$ \\
\hline$C(5)$ & $2 i$ & $0.4073(3)$ & $0.1477(3)$ & $-0.0495(2)$ & $0.064(2)$ & $0.052(1)$ & $0.049(1)$ & $0.033(1)$ & $0.019(1)$ & $0.019(1)$ \\
\hline$C(6)$ & $2 i$ & $0.4286(3)$ & $0.0946(3)$ & $-0.1674(2)$ & $0.072(2)$ & $0.062(2)$ & $0.068(2)$ & $0.045(1)$ & $0.030(1)$ & $0.025(1)$ \\
\hline$C(8)$ & $2 i$ & $0.1946(2)$ & $0.3695(2)$ & $-0.1798(2)$ & $0.033(1)$ & $0.035(1)$ & $0.032(1)$ & $0.0124(9)$ & $0.0124(9)$ & $0.0084(9)$ \\
\hline $\mathrm{C}(9)$ & $2 i$ & $0.0251(2)$ & $0.2856(2)$ & $-0.2144(2)$ & $0.037(1)$ & $0.039(1)$ & $0.041(1)$ & $0.0156(9)$ & $0.018(1)$ & $0.016(1)$ \\
\hline$C(10)$ & $2 i$ & $-0.1122(2)$ & $0.1931(3)$ & $-0.3407(2)$ & $0.036(1)$ & $0.060(2)$ & $0.045(1)$ & $0.011(1)$ & $0.012(1)$ & $0.015(1)$ \\
\hline$C(11)$ & $2 i$ & $-0.2624(3)$ & $0.1475(3)$ & $-0.3346(3)$ & $0.038(1)$ & $0.076(2)$ & $0.060(2)$ & $0.014(1)$ & $0.015(1)$ & $0.008(1)$ \\
\hline$C(12)$ & $2 i$ & $-0.2971(3)$ & $0.0290(3)$ & $-0.2736(3)$ & $0.064(2)$ & $0.061(2)$ & $0.094(2)$ & $0.006(1)$ & $0.052(2)$ & $0.005(2)$ \\
\hline$C(13)$ & $2 i$ & $-0.1736(3)$ & $0.0419(3)$ & $-0.1429(3)$ & $0.087(2)$ & $0.056(2)$ & $0.112(3)$ & $0.029(2)$ & $0.071(2)$ & $0.042(2)$ \\
\hline$C(14)$ & $2 i$ & $-0.1256(3)$ & $0.1595(3)$ & $-0.0238(3)$ & $0.071(2)$ & $0.080(2)$ & $0.076(2)$ & $0.027(2)$ & $0.047(2)$ & $0.041(2)$ \\
\hline$C(17)$ & $2 i$ & $0.2039(2)$ & $0.4218(2)$ & $-0.0423(2)$ & $0.041(1)$ & $0.039(1)$ & $0.039(1)$ & $0.021(1)$ & $0.017(1)$ & $0.014(1)$ \\
\hline$O(17)$ & $2 i$ & $0.3071(2)$ & $0.5025(2)$ & $0.0619(1)$ & $0.0479(9)$ & $0.060(1)$ & $0.0391(9)$ & $0.0181(8)$ & $0.0142(8)$ & $0.0022(8)$ \\
\hline$C(21)$ & $2 i$ & $0.2124(3)$ & $0.2385(2)$ & $-0.4185(2)$ & $0.044(1)$ & $0.042(1)$ & $0.038(1)$ & $0.017(1)$ & $0.018(1)$ & $0.006(1)$ \\
\hline$O(21)$ & $2 i$ & $0.0763(2)$ & $0.1847(2)$ & $-0.4660(2)$ & $0.043(1)$ & $0.078(1)$ & $0.0443(9)$ & $0.0154(9)$ & $0.0155(8)$ & $0.0176(9)$ \\
\hline $\mathrm{C}(22)$ & $2 i$ & $0.2852(3)$ & $0.3005(3)$ & $-0.5021(2)$ & $0.050(1)$ & $0.063(2)$ & $0.040(1)$ & $0.023(1)$ & $0.021(1)$ & $0.012(1)$ \\
\hline$O(22)$ & $2 i$ & $0.4029(2)$ & $0.3054(2)$ & $-0.4910(2)$ & $0.075(1)$ & $0.132(2)$ & $0.079(1)$ & $0.058(1)$ & $0.053(1)$ & $0.052(1)$ \\
\hline$O(23)$ & $2 i$ & $0.2001(2)$ & $0.3433(2)$ & $-0.5887(2)$ & $0.061(1)$ & $0.109(2)$ & $0.059(1)$ & $0.037(1)$ & $0.0291(9)$ & $0.048(1)$ \\
\hline$C(24)$ & $2 i$ & $0.2587(4)$ & $0.4056(4)$ & $-0.6721(3)$ & $0.083(2)$ & $0.154(3)$ & $0.081(2)$ & $0.041(2)$ & $0.041(2)$ & $0.074(2)$ \\
\hline$C(81)$ & $2 i$ & $0.2277(2)$ & $0.4875(2)$ & $-0.2418(2)$ & $0.038(1)$ & $0.033(1)$ & $0.032(1)$ & $0.0140(9)$ & $0.0129(9)$ & $0.0079(9)$ \\
\hline$C(82)$ & $2 i$ & $0.3644(2)$ & $0.5498(2)$ & $-0.2464(2)$ & $0.041(1)$ & $0.039(1)$ & $0.047(1)$ & $0.014(1)$ & $0.018(1)$ & $0.015(1)$ \\
\hline$C(83)$ & $2 i$ & $0.3932(3)$ & $0.6590(2)$ & $-0.3015(2)$ & $0.054(1)$ & $0.044(1)$ & $0.057(1)$ & $0.012(1)$ & $0.025(1)$ & $0.019(1)$ \\
\hline$C(84)$ & $2 i$ & $0.2879(3)$ & $0.7093(3)$ & $-0.3502(2)$ & $0.067(2)$ & $0.045(1)$ & $0.053(1)$ & $0.019(1)$ & $0.021(1)$ & $0.024(1)$ \\
\hline$C(85)$ & $2 i$ & $0.1530(3)$ & $0.6507(3)$ & $-0.3460(2)$ & $0.062(2)$ & $0.056(2)$ & $0.066(2)$ & $0.034(1)$ & $0.021(1)$ & $0.030(1)$ \\
\hline$C(86)$ & $2 i$ & $0.1235(3)$ & $0.5413(2)$ & $-0.2914(2)$ & $0.049(1)$ & $0.051(1)$ & $0.058(1)$ & $0.026(1)$ & $0.024(1)$ & $0.023(1)$ \\
\hline
\end{tabular}

\section{References}

1. Christl, M.; Lanzendörfer, U.; Grötsch, M. M.; Hegmann, J.: Cycloadditions of 1,3,4-oxadiazin-6-ones (4,5-diaza- $\alpha$-pyrones). 4. Primary adducts, $\gamma$-oxo ketenes and several products of the reaction of methyl 6-oxo-5-phenyl-1,3,4-oxadiazine-2-carboxylate with norbornene. Angew. Chem. Int. Ed. Engl. 24 (1985) 886-887.

2. Sheldrick, G. M.: Program Package SHELXTL-plus. Release 4.1. Siemens Analytical X-Ray Instruments Inc., Madison (WI 53719), USA 1990. 